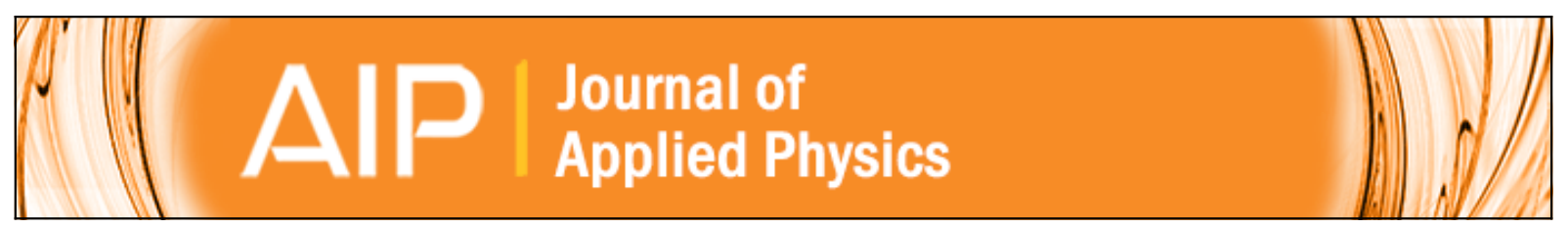

\title{
Random laser action from flexible biocellulose-based device
}

Molíria V. dos Santos, Christian T. Dominguez, João V. Schiavon, Hernane S. Barud, Luciana S. A. de Melo, Sidney J. L. Ribeiro, Anderson S. L. Gomes, and Cid B. de Araújo

Citation: Journal of Applied Physics 115, 083108 (2014); doi: 10.1063/1.4866686

View online: http://dx.doi.org/10.1063/1.4866686

View Table of Contents: http://scitation.aip.org/content/aip/journal/jap/115/8?ver=pdfcov

Published by the AIP Publishing

\section{Articles you may be interested in}

Accelerated Escherichia coli inactivation in the dark on uniform copper flexible surfaces

Biointerphases 9, 029012 (2014); 10.1116/1.4870596

Random laser action with nanostructures in a dye solution

J. Laser Appl. 25, 042012 (2013); 10.2351/1.4809615

A flexible organic random laser based on poly(9,9-dioctylfluorene) deposited on a surface corrugated polyphthalate-carbonate substrate

Appl. Phys. Lett. 98, 253304 (2011); 10.1063/1.3601854

Random laser action in dye solutions containing Stöber silica nanoparticles

J. Appl. Phys. 108, 033508 (2010); 10.1063/1.3462443

Random lasing following two-photon excitation of highly scattering gain media Appl. Phys. Lett. 81, 2511 (2002); 10.1063/1.1511284

\section{AIP $\mid$ chaos CALL FOR APPLICANTS Seeking new Editor-in-Chief}




\title{
Random laser action from flexible biocellulose-based device
}

\author{
Molíria V. dos Santos, ${ }^{1}$ Christian T. Dominguez, ${ }^{2,3, a)}$ João V. Schiavon, ${ }^{1}$ Hernane S. Barud, ${ }^{1}$ \\ Luciana S. A. de Melo, ${ }^{3}$ Sidney J. L. Ribeiro, ${ }^{1}$ Anderson S. L. Gomes, ${ }^{2}$ \\ and Cid B. de Araújo ${ }^{2}$ \\ ${ }_{1}^{1}$ Instituto de Química, Universidade do Estado de São Paulo (UNESP), CP 355, Araraquara, \\ SP 14801-970, Brazil \\ ${ }^{2}$ Departamento de Física, Universidade Federal de Pernambuco, Recife, PE 50670-901, Brazil \\ ${ }^{3}$ Laboratório de Óptica Biomédica e Imagem, Universidade Federal de Pernambuco, Recife, \\ PE 50740-530, Brazil
}

(Received 18 November 2013; accepted 11 February 2014; published online 26 February 2014)

\begin{abstract}
We demonstrate random lasing action in flexible bacterial cellulose (BC) membrane containing a laser-dye and either dielectric or metallic nanoparticles (NPs). The novel random laser system consists of $\mathrm{BC}$ nanofibers attached with Rhodamine 6G molecules and having incorporated either silica or silver NPs. The laser action was obtained by excitation of the samples with a 6 ns pulsed laser at $532 \mathrm{~nm}$. Minimum laser threshold of $\approx 0.7 \mathrm{~mJ} /$ pulse was measured for the samples with silica NPs, whereas a laser threshold of $2.5 \mathrm{~mJ} /$ pulse for a system based on silver NPs was obtained. In both cases a linewidth narrowing from $\approx 50$ to $\approx 4 \mathrm{~nm}$ was observed. Potential applications in biophotonics and life sciences are discussed for this proof-of-concept device. (C) 2014 AIP Publishing LLC. [http://dx.doi.org/10.1063/1.4866686]
\end{abstract}

\section{INTRODUCTION}

The multidisciplinary field of biophotonics, which deals with photonic applications in biology and bio-related areas, has received increased attention in recent years. ${ }^{1}$ Among important developments, the observation of Random Laser (RL) emission in biomaterials have been reported since the early $1990 \mathrm{~s}^{2,3}$ with recent advances leading to the demonstration of a single cell biological laser. ${ }^{4,5}$ RLs are a special kind of lasers in which the optical feedback is due to light scattering in amplifying media instead of a conventional Fabry-Perot cavity, ${ }^{6}$ as reviewed in Refs. 7-11. After the demonstration by Lawandy et al. ${ }^{12}$ of RLs based on dye solutions containing $\mathrm{TiO}_{2}$ nanoparticles (NPs), there has been a rapid growth in this field and RL action has been observed in a large variety of systems such as dye doped polymer films, ${ }^{13}$ semiconductor powders, ${ }^{14,15}$ organic-inorganic hybrids, ${ }^{16}$ and optical fibers. ${ }^{17}$ From the biophotonics point of view, RLs in biomaterials have been reported in Refs. 18-21. Recently Viola et $a{ }^{22}{ }^{22}$ demonstrated RL action of a device based on common and commercial paper using conventional soft-lithography techniques to create porous channels on the cellulose fiber. This work shows the high flexibility of paper is the way for innovative applications of RL devices.

The motivation for the present work was to demonstrate, as a proof-of-concept device, RL action in a host biomaterial, biocellulose, or bacterial cellulose (BC), which by itself has already been exploited in biophotonics and medicine. ${ }^{23,24}$ $\mathrm{BC}$ is a biocompatible material produced by the bacteria Gluconacetobacter xylinus, with a three-dimensional network structure composed of microfibrils having nanometric diameters. Supramolecular hydrogen bonds involving the fibrillar units stabilize the whole structure and give large

\footnotetext{
a) Author to whom correspondence should be addressed. Electronic mail: christian@df.ufpe.br
}

mechanical strength. ${ }^{23}$ In the never dried state, BC membranes present relatively high porosity allowing the incorporation or synthesis in situ of silica $\left(\mathrm{SiO}_{2}\right)^{25}$ or metallic $\mathrm{NPs}^{26}$ The large potential of BC for applications in biomedicine has been demonstrated as small-diameter blood vessels replacement ${ }^{27}$ and as temporary skin substitute in the treatment of chronic wounds, burns, and ulcers. ${ }^{28}$ The incorporation of silver (Ag) NPs as antimicrobial body extended the biomedical applications of BC membranes. ${ }^{26}$

The herein reported RL was fabricated using a flexible biocellulose membranes as host matrices and as the gain medium, for concept proof, molecules of Rhodamine $6 \mathrm{G}$ (Rh6G) were attached to BC microfibrils. Silica or Ag NPs were incorporated to the $\mathrm{BC}$ membranes to act as the scattering medium responsible for optical feedback. The obtained materials are flexible and present typical RL characteristics such as emitted nonlinear intensity dependence with the excitation intensity, large spectral bandwidth narrowing with the increase of the excitation intensity and excitation threshold behavior.

\section{EXPERIMENTAL DETAILS}

BC membranes containing silica NPs were produced according to the procedure described in Ref. 29. Silica NPs were chosen as scatterers because the photodegradation of the laser-dye molecules is smaller than it is observed using $\mathrm{TiO}_{2}$ NPs when excitation lasers operating in the green range are used. ${ }^{30}$ Briefly, BC-silica hybrids were prepared from the hydrolysis/condensation of tetraethoxysilane (TEOS) on the cellulose microfibrils. Never dried BC membranes (dimensions: $50 \times 40 \times 0.3 \mathrm{~mm}^{3}$ ) were immersed in $25 \mathrm{ml}$ of a solution containing ethanol/TEOS at different concentrations of TEOS/ethanol during 10 days. Therefore, membranes containing $\mathrm{SiO}_{2}$ NPs (diameters: $\approx 25 \mathrm{~nm}$ ) attached to microfibrils of $\mathrm{BC}$ were obtained. 
Ag NPs were also used due to its ability to act as scatterers and due to the excitation of surface plasmons (SPs) that induces optical properties hardly achievable in other optical materials. Furthermore, its antimicrobial activity potentializes biophotonic applications. ${ }^{31,32}$ To synthesize the Ag NPs, $20 \mathrm{ml}$ of aqueous solution containing $\mathrm{AgNO}_{3}(0.25 \mathrm{mM})$ and sodium citrate $(0.25 \mathrm{mM})$ were prepared. Then, a solution of $0.6 \mathrm{ml}$ of $\mathrm{NaBH}_{4}(0.1 \mathrm{M})$, which had been chilled in an icebath, was added. The final solution remained under strong stirring for $3 \mathrm{~h}$ to complete the reaction. The samples for the experiments were obtained immersing the $\mathrm{BC}$ membranes for $24 \mathrm{~h}$ in $10 \mathrm{ml}$ of a colloid containing Ag NPs. After this procedure, the samples were submitted to a lyophilization for $24 \mathrm{~h}$

To infiltrate the gain medium, the $\mathrm{BC}$ membranes were immersed in $10 \mathrm{ml}$ of an ethanol solution containing $10^{-4} \mathrm{M}$ of Rh6G and kept under strong stirring during $24 \mathrm{~h}$ at room temperature. Finally, the membranes were removed from the dye solution and placed in an oven at $40^{\circ} \mathrm{C}$, during $2 \mathrm{~h}$, for evaporation of the ethanol.

RL emission was achieved focusing the second harmonic of a pulsed Nd:YAG laser beam $(532 \mathrm{~nm}, 6 \mathrm{~ns})$ on the membrane's face of each sample, with a $3 \mathrm{~mm}$ diameter laser beam spot. Single laser-shot luminescence signals were detected in a direction of $\approx 45^{\circ}$ with respect to the membrane surface. The emission spectra were analyzed using a CCD coupled monochromator with $0.1 \mathrm{~nm}$ spectral resolution.

\section{RESULTS AND DISCUSSIONS}

To characterize the $\mathrm{BC}$ membranes after the hydrolysis/ condensation process, micrographs of scanning electronic microscopy (SEM) were obtained. Fig. 1 shows a SEM image of a $\mathrm{BC}$-silica membrane with mass percentage ratio of 37\% (sample: BC-silica 37\%). Spherical silica NPs are observed attached to the network formed by $\mathrm{BC}$ microfibrils. ${ }^{29}$ From the SEM images, we deduced that the membranes play the role of a tridimensional network to support the silica NPs. Additionally, no changes in the morphology or microstructure of $\mathrm{BC}$ membranes are observed after the synthesis.

The amount of silica in the $\mathrm{BC}$ membranes was estimated performing thermogravimetric analysis (TGA). As

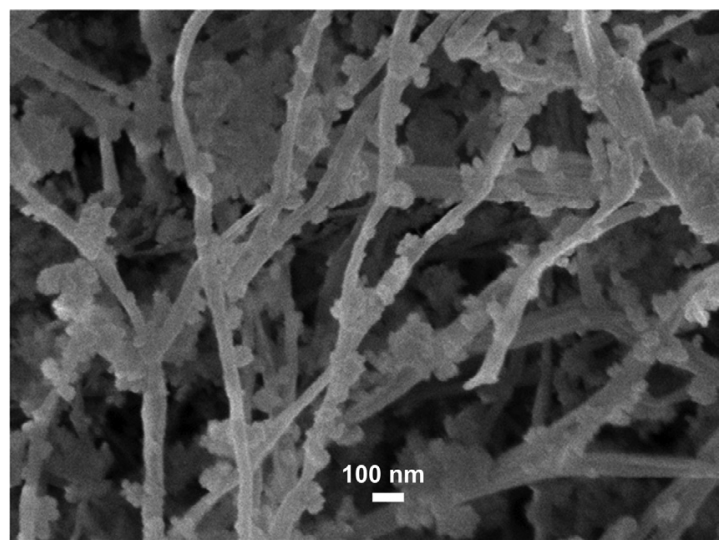

FIG. 1. SEM image of the cellulose membrane (sample: BC-silica 37\%). soon as TGA curve was performed in an oxygen atmosphere the final product is attributed to silica nanoparticles, Fig. 2(a). Curve (i) corresponds to a BC membrane without silica NPs neither Rh6G. It is observed a mass loss of $\approx 5 \%$ between 80 and $100{ }^{\circ} \mathrm{C}$ due to evaporation of water that was remaining in the surface of the membrane. Two stages associated to thermal degradation of the $\mathrm{BC}$ membrane are observed at $\approx 330^{\circ} \mathrm{C}$ and $\approx 460^{\circ} \mathrm{C}$. At this temperature range, processes such as depolymerization, dehydration, and breakdown of glycosidic bonds and the subsequent formation of $\mathrm{CO}_{2}$ and water are observed. ${ }^{29}$ Similarly, curves (iii) and (iv) for the BC-silica samples show a first stage of superficial water evaporation from the membrane between 80 and $100{ }^{\circ} \mathrm{C}$ followed by two stages of $\mathrm{BC}$ decomposition. It is observed that the thermal stability of the $\mathrm{BC}$ membranes around $300^{\circ} \mathrm{C}$ is increased with addition of the silica NPs, which improve the stability of RL emission, minimizing thermal damage due to high pumping fluences.

Figure 2(b) shows the Fourier Transformed Infrared (FTIR) spectra of five samples. The main bands associated to each spectrum are similar to the ones described in Ref. 29. Each spectrum can be considered as the superimposed spectrum of the $\mathrm{BC}$ and amorphous silica weighted by the relative concentrations of the two components. For example, due to the silica NPs, the bands at $460 \mathrm{~cm}^{-1} \delta$ (Si-O-Si),
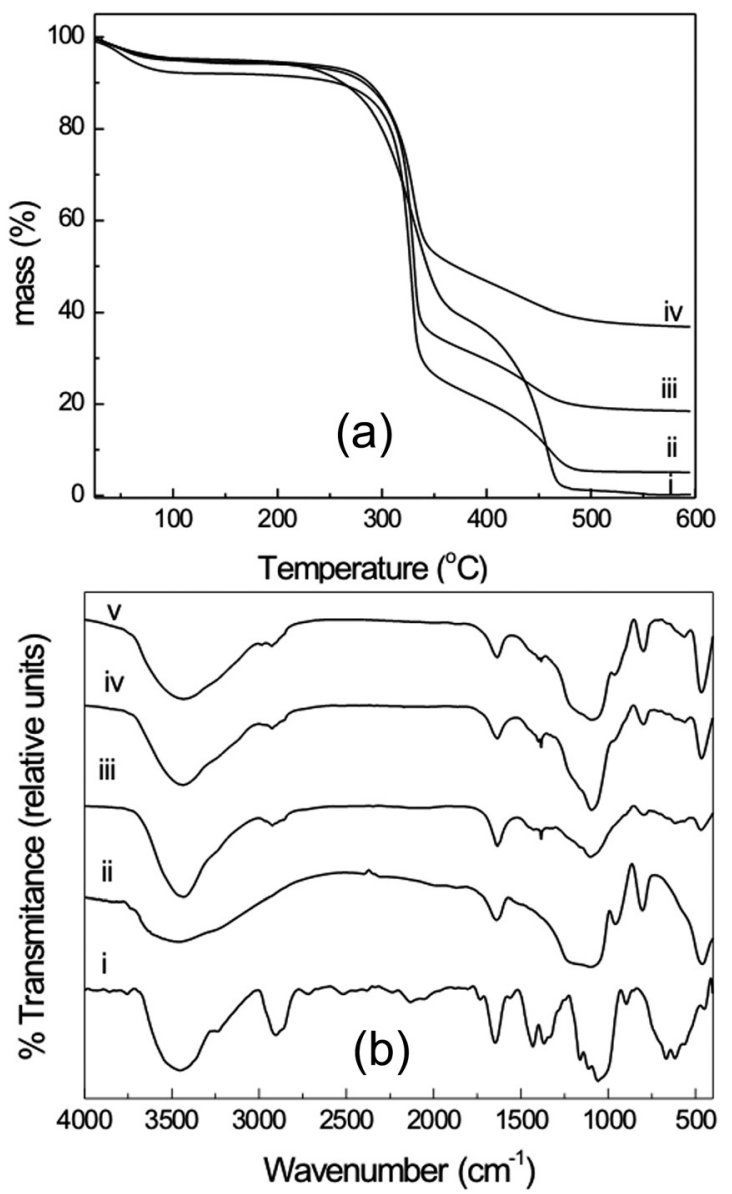

FIG. 2. (a) TGA curves corresponding to: (i) Bacterial cellulose, (ii) sample: BC-silica 5\%, (iii) sample: BC-silica 18\%, and (iv) sample: BC-silica 37\%. (b) FTIR spectra of (i) bacterial cellulose, (ii) amorphous silica, (iii) sample: BC-silica 5\%, (iv) sample: BC-silica 18\%, and (v) sample: BC-silica 37\%. 
$785 \mathrm{~cm}^{-1} \nu \mathrm{s}(\mathrm{Si}-\mathrm{O}-\mathrm{Si}), 940 \mathrm{~cm}^{-1} \nu(\mathrm{Si}-\mathrm{OH})$, and $1085 \mathrm{~cm}^{-1}$ $\nu \mathrm{s}(\mathrm{Si}-\mathrm{O}-\mathrm{Si})$ are enhanced. The results for the hybrid samples (curves iii-v) show a decrease of the amplitudes related to $\mathrm{BC}$ bands at $2900 \mathrm{~cm}^{-1}\left(\nu \mathrm{CH}_{2}\right), 2700 \mathrm{~cm}^{-1}\left(\nu_{\mathrm{a}} \mathrm{CH}_{2}\right)$, $1400 \mathrm{~cm}^{-1}\left(\delta \mathrm{CH}_{2}\right)$, and $1370 \mathrm{~cm}^{-1}\left(\delta \mathrm{CH}_{3}\right)$, in comparison with curve (i). Additionally, a broadening of the $3500 \mathrm{~cm}^{-1}$ $(\nu \mathrm{O}-\mathrm{H})$ band is observed. These spectral changes may be due to hydrogen bonds formation between hydroxyls groups from bacterial cellulose and the silica nanoparticles. ${ }^{29}$

For the samples containing Ag NPs, another characterization procedure was employed. Fig. 3(a) shows the linear optical absorption spectrum of a colloidal solution containing Ag NPs. The well-known surface plasmons resonance due to spherical NPs is centered at $\approx 400 \mathrm{~nm}$. The shape and size of Ag NPs were determined from transmission electron microscopy (TEM) images as shown in Fig. 3(b). The size distribution of the Ag NPs, shown in Fig. 3(c), presents an average diameter $(9.3 \pm 0.2) \mathrm{nm}$; the histogram was obtained by measuring $\approx 400$ particles. Fig. 3(c) shows that the $\mathrm{Ag}$ NPs have spherical shape, and Fig. 3(d) shows a TEM image of a BC membrane containing Ag NPs. As in the silica NPs case, the Ag NPs are attached to the BC fibrils.

Shown in Fig. 4(a) is a photo-image of a BC membrane containing Rh6G and silica NPs; the BC membrane-based RL has a paper-like appearance and is totally flexible, which facilitates its manipulation.

Fig. 4(b) displays the luminescence of a $\mathrm{BC}$ membrane containing only Rh6G while Fig. 4(c) shows the emission of the BC-silica 18\% sample having the same Rh6G amount. It is important to note that the samples were excited using the same laser intensity and focal spot but there is a large difference in the light intensity emitted by the two samples. The luminescence of the $\mathrm{BC}$ sample is due to $\mathrm{Rh} 6 \mathrm{G}$ dye alone and the larger luminescence of sample BC-silica is the RL

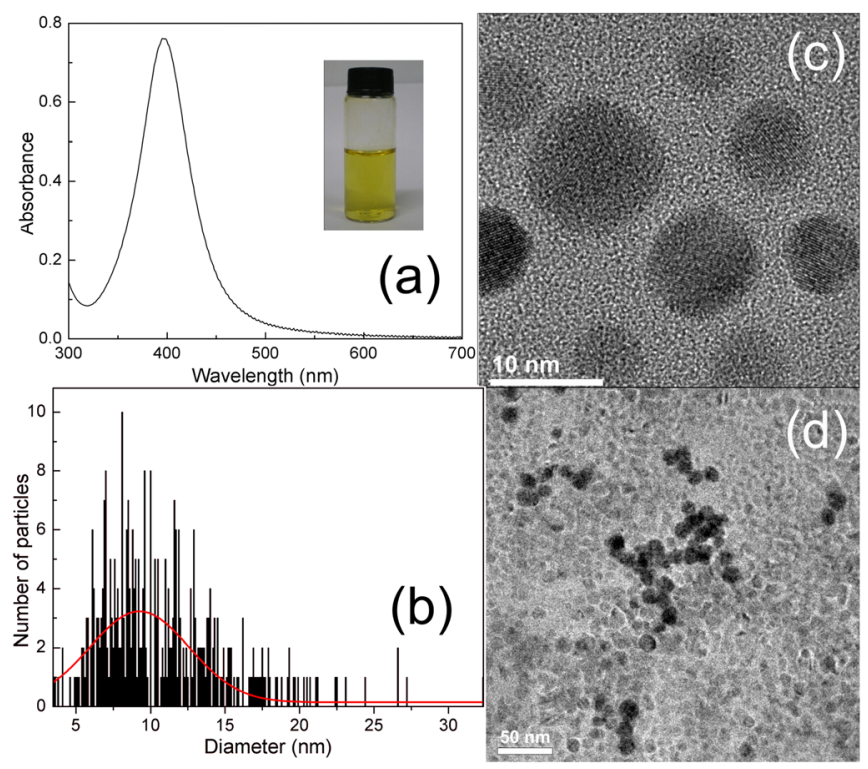

FIG. 3. (a) UV-Vis spectrum of Ag NPs in colloidal solution. (b) Size distribution of Ag NPs in colloidal solution obtained using the DLS technique. (c) TEM image of Ag NPs in colloidal solution. The image inset in (a) is a photograph of the colloidal solution containing Ag NPs. (d) TEM image of a BC membrane containing Ag NPs.

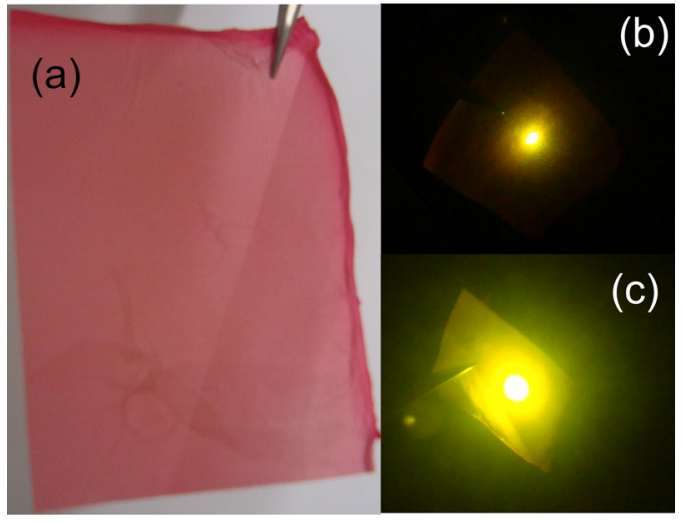

FIG. 4. (a) Photo-image of a BC membrane containing silica NPs. (b) Luminescence of Rh6G in a BC membrane without NPs. (c) Random laser emission from sample BC-silica $18 \%$. The pump energy was $3.52 \mathrm{~mJ}$ per pulse in both photographed emissions.

emission due to the optical feedback provided by the silica NPs. To obtain the luminescence images, a green filter was used in front of a digital camera to cut-off the specular scattered $532 \mathrm{~nm}$ laser light. RL emission was not observed from BC membranes containing only Rh6G dye probably because the light scattering due to the fibrils is not efficient enough to provide the required optical feedback for RL action.

In order to further characterize and confirm the RL emission, the emitted intensity behavior and bandwidth narrowing as a function of pump energy were measured, as shown in Figs. 5(a) and 5(b), respectively. The plot of the peak intensity versus the pump energy corresponding to samples BC-silica 18\% (green stars) and BC silica 37\% (black circles) shows a strong nonlinear behavior that is characteristic of RL action, whereas at 5\% (inset in Fig. 5(a)), the nonlinear emission intensity behavior is not yet clearly defined. The linewidth narrowing as a function of the laser pulse energy is typical of RL based on laser-dyes and clarifies the laser threshold; the linewidth reaches $\approx 4 \mathrm{~nm}$ for the samples BC-silica $18 \%$ and BC-silica $37 \%$ at high excitation intensity, and about $10 \mathrm{~nm}$ for BC-silica 5\%. The energy thresholds were determined considering the pumping energy per pulse corresponding to half of the maximum linewidth. The values obtained were $1.9 \mathrm{~mJ} /$ pulse (BC-silica $5 \%$ ), $0.72 \mathrm{~mJ} /$ pulse (BC-silica $18 \%$ ), and $0.71 \mathrm{~mJ} /$ pulse (BC-silica $37 \%$ ). In order to show the threshold dependence as function of number of NPs presents in the cellulose membrane, we present in Table I the values of NPs density of samples used in this experiment.

As mentioned before, we also investigated $\mathrm{BC}$ membranes containing Rh6G and Ag NPs. RLs using Ag NPs as responsible for enhanced emission was reported before with poly(methylmethacrylate) - PMMA — as the host medium ${ }^{13}$ or other polymers, ${ }^{33,34}$ whereby even in the weakly scattering regime arising from the NPs smaller size and crosssection, coherent RL emission has been observed. ${ }^{34}$ In the present case, the Ag NPs were introduced in the BC membrane as described in Sec. II. Fig. 6 shows the spectral evolution for different pumping energies, the behavior of the peak intensity and the dependence of the emission linewidth as a function of pumping energy for the sample having mass 

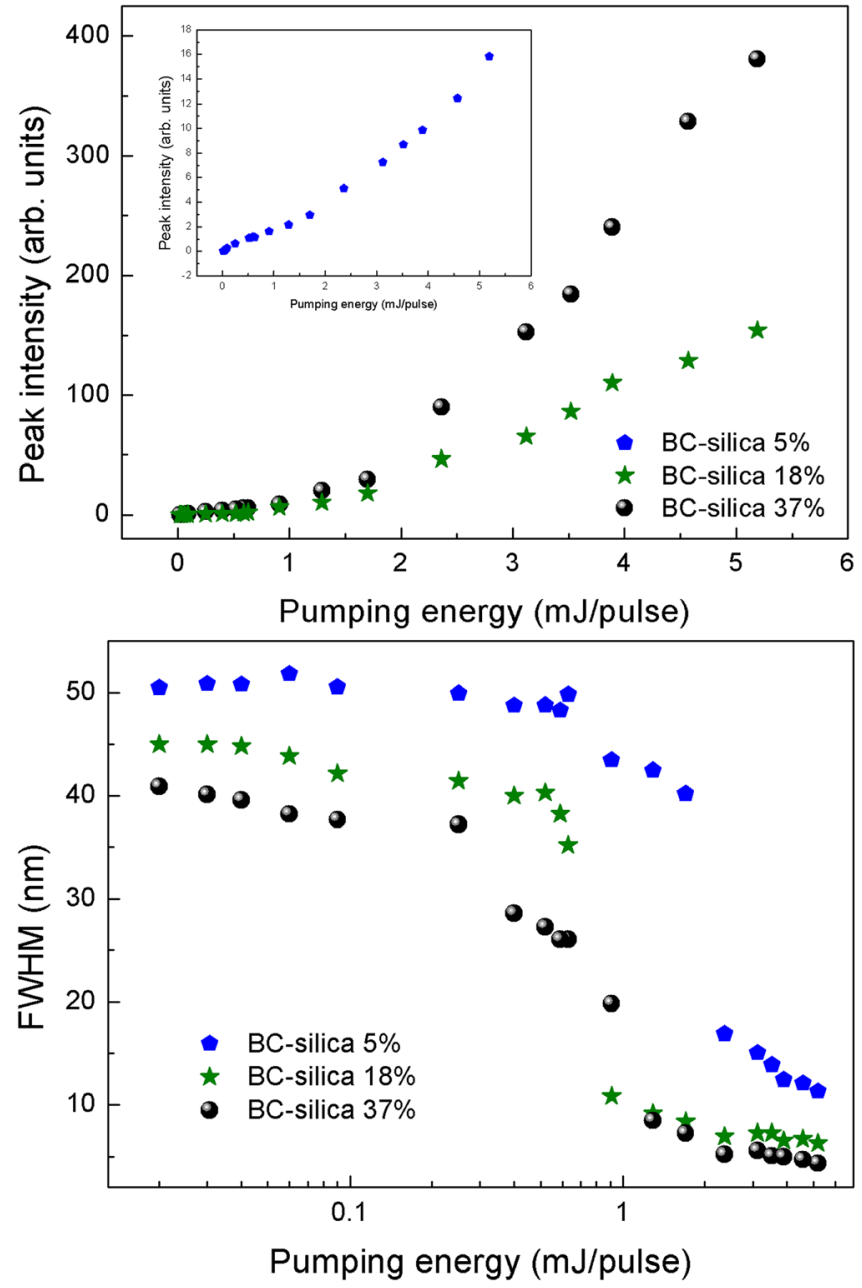

FIG. 5. (a) Peak intensity vs. pumping energy per pulse showing the nonlinear behavior of three samples containing different silica concentrations (inset: sample BC-silica 5\%). (b) Full width half maximum (FWHM) linewidth vs. pumping energy per pulse. The minimum linewidth was $\approx 4 \mathrm{~nm}$ for pumping above the threshold.

percentage ratio of silver of $0.1 \%$ correspondent to $2.4 \times 10^{13} \mathrm{~cm}^{-3}$ (see Table I). In this case, the laser threshold was estimated to be $2.5 \mathrm{~mJ} /$ pulse, determined as for the samples with silica particles, and coincident with the value where nonlinear behavior of emitted intensity starts (see Fig. 6(c)).

The linewidth behavior in the BC-Ag NPs system is about the same reported in Ref. 13 and the RL threshold is of the same order of magnitude. In RL with metallic NPs besides the feedback mechanism due to multiple scattering of light, the local field in the vicinity of the NPs is large and then the molecules located nearby the NPs present larger

TABLE I. Values of NPs densities for silica and Ag in the BC membranes.

\begin{tabular}{lcccc}
\hline \hline & \multicolumn{2}{c}{ Silica NPs } & \multicolumn{2}{c}{ Ag NPs } \\
\cline { 1 - 1 }$\%(\mathrm{~m} / \mathrm{m})$ & Density $\left(\times 10^{15} \mathrm{~cm}^{-3}\right)$ & & $\%(\mathrm{~m} / \mathrm{m})$ & Density $\left(\times 10^{13} \mathrm{~cm}^{-3}\right)$ \\
\hline 5 & 0.4 & 0.1 & 2.4 \\
18 & 1.5 & & \\
37 & 3.1 & & \\
\hline \hline
\end{tabular}
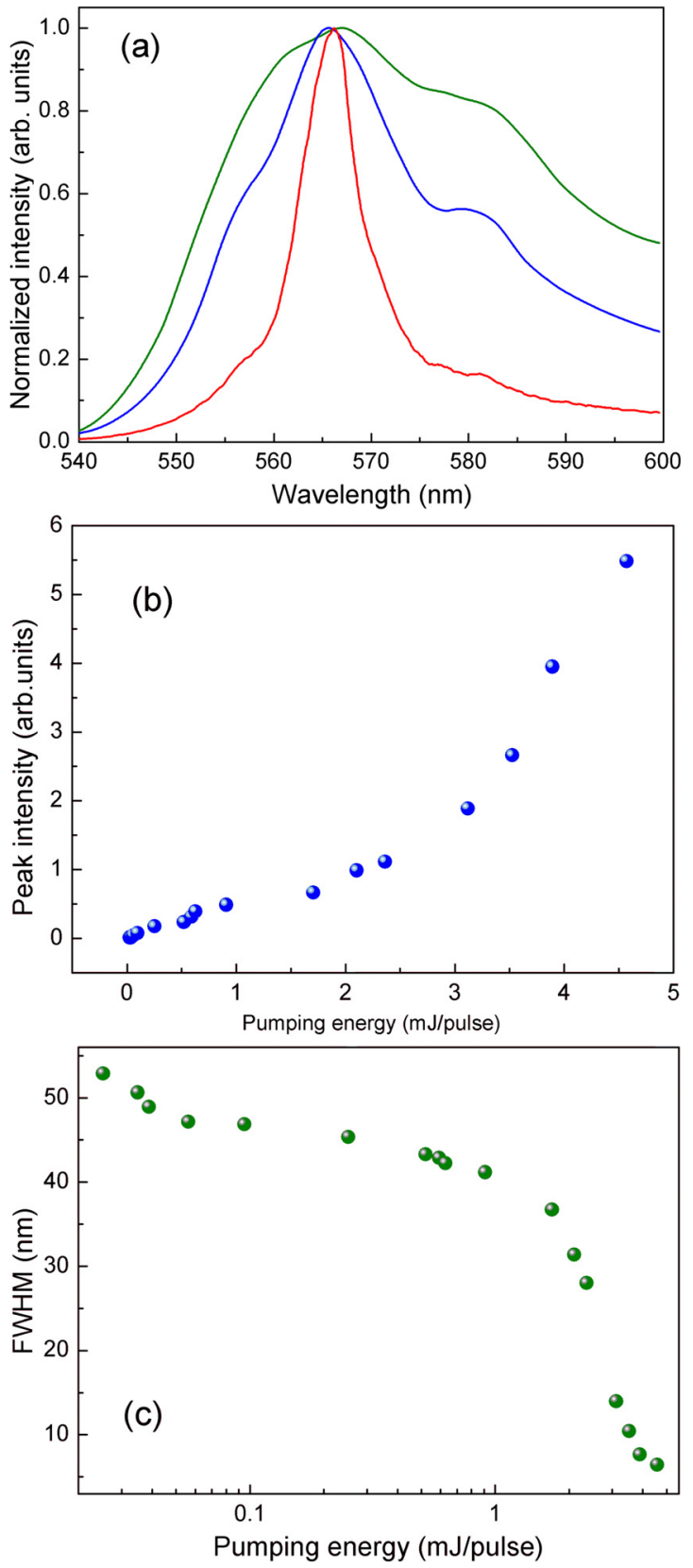

FIG. 6. Spectral behavior of the BC membrane containing Rh6G and Ag NPs. (a) Spectrum of the RL for excitation pulses of $0.6 \mathrm{~mJ}$ (green line), $2.4 \mathrm{~mJ}$ (blue line), and $3.9 \mathrm{~mJ}$ (red line). (b) Peak intensity behavior and (c) FWHM linewidth vs. the pumping energy per pulse. Minimum linewidth observed is about $5 \mathrm{~nm}$.

radiative transition probability than the molecules far from the metallic particles. Hence, the RL emission may be dominated by the molecules positioned around the NPs. Similar to the case of the BC-silica NPs as well as in Ref. 13, the RL wavelength was centered at $\approx 567 \mathrm{~nm}$, near the maximum of the luminescence band.

Other samples with larger concentration of silver were investigated but RL emission was not observed probably because the Rh6G molecules tend to bind on the Ag NPs to form Ag NP-Rh6G complexes. In such cases energy transfer from the molecule to the NP is enhanced and competes with the stimulated emission mechanism resulting in quenching of the RL process. This behavior was also observed in 
Ref. 13 and its presence certainly deserves more investigation in the future.

\section{CONCLUSIONS}

We demonstrated efficient RL action in flexible biocellulose-based randomly infiltrated with Rh6G and containing silica or Ag NPs. The addition of the NPs in the BC membranes allows observation of the RL phenomenon that cannot be otherwise observed. From the spectral behavior, it is inferred the RL operates in the diffusive regime with either type of NPs. ${ }^{7}$ This work opens the possibility of new applications of the RL phenomenon in photodynamic therapy and dermatology where light with a narrow linewidth is more appropriate to activate the biophotonic processes that can be exploited for the treatment of superficial lesions. It is still more exciting when related to the already demonstrated RL emission in $\operatorname{skin}^{18}$ and bone tissue, ${ }^{20}$ where the scattering media is the tissue. Embedding BC in tissue has been demonstrated to help healing; therefore, the use of the platform BC-dye-NPs can be used for diagnostics or, if Ag NP is employed, could be used for improving antibacterial processes. Using Coumarin dyes that are biocompatible and have already been used in $\mathrm{RLs}^{35}$ will further potentialize the concept-device reported here. Further applications arise from the fact that, since the $\mathrm{BC}$ based RL operates in the incoherent feedback regime, it can be potentially used for specklefree laser imaging. ${ }^{36}$

\section{ACKNOWLEDGMENTS}

This work was supported by the Conselho Nacional de Desenvolvimento Científico e Tecnológico (CNPq), through the National Institute of Photonics (INCT de Fotônica) and by a joint grant from CNPq and FACEPE (Fundação de Amparo à Ciência e Tecnologia do Estado de Pernambuco) through the PRONEX program. We also thank FAPESP (Fundação de Amparo à Pesquisa do Estado de São Paulo) and the LME/LNNano/CNPEM for technical support.

${ }^{1}$ P. N. Prasad, Introduction to Biophotonics (Wiley, New York, 2003).

${ }^{2}$ M. Siddique, L. Yang, Q. Z. Wang, and R. R. Alfano, Opt. Commun. 117, 475 (1995).

${ }^{3}$ L. Wang, D. Liu, N. He, S. L. Jacques, and S. L. Thomsen, Appl. Opt. 35, 1775 (1996).
${ }^{4}$ M. C. Gather and S. H. Yun, Nature Photon. 5, 406 (2011).

${ }^{5}$ M. C. Gather and S. H. Yun, Opt. Lett. 36, 3299 (2011).

${ }^{6}$ V. S. Letokhov, Sov. Phys. JETP 26, 835 (1968).

${ }^{7}$ H. Cao, Waves Random Media 13, R1 (2003)

${ }^{8}$ D. S. Wiersma, Nat. Phys. 4, 359 (2008).

${ }^{9}$ M. A. Noginov, Solid-State Random Lasers (Springer, New York, 2005).

${ }^{10}$ D. S. Wiersma and M. A. Noginov, J. Opt. 12, 020201 (2010).

${ }^{11}$ D. S. Wiersma, Nature Photon. 7, 188 (2013).

${ }^{12}$ N. M. Lawandy, R. M. Balachandran, A. S. L. Gomes, and E. Sauvain, Nature 368, 436 (1994).

${ }^{13}$ C. Tolentino Dominguez, R. L. Maltez, R. M. S. dos Reis, L. S. A. de Melo, C. B. de Araújo, and A. S. L. Gomes, J. Opt. Soc. Am. B 28, 1118 (2011).

${ }^{14}$ H. Cao, Y. Zhao, S. T. Ho, E. W. Seelig, Q. H. Wang, and R. P. H. Chang, Phys. Rev. Lett. 82, 2278 (1999).

${ }^{15}$ G. Zhu, C. E. Small, and M. A. Noginov, Opt. Lett. 33, 920 (2008).

${ }^{16}$ E. Pecoraro, S. García-Revilla, R. A. S. Ferreira, R. Balda, L. D. Carlos, and J. Fernández, Opt. Express 18, 7470 (2010).

${ }^{17}$ C. J. S. de Matos, L. de S. Menezes, A. M. Brito-Silva, M. A. M. Gamez, A. S. L. Gomes, and C. B. de Araújo, Phys. Rev. Lett. 99, 153903 (2007).

${ }^{18}$ R. C. Polson and Z. V. Vardeny, Appl. Phys. Lett. 85, 1289 (2004).

${ }^{19}$ R. C. Polson and Z. V. Vardeny, J. Opt. 12, 024010 (2010).

${ }^{20}$ Q. Song, S. Xiao, Z. Xu, J. Liu, X. Sun, V. Drachev, V. M. Shalaev, O. Akkus, and Y. I. Kim, Opt. Lett. 35, 1425 (2010).

${ }^{21}$ D. Zhang, G. Kostovski, C. Karnutsch, and A. Mitchell, Org. Electron. 13, 2342 (2012).

${ }^{22}$ I. Viola, N. Ghofraniha, A. Zacheo, V. Arima, C. Conti, and G. Gigli, J. Mater. Chem. C 1, 8128 (2013).

${ }^{23}$ W. K. Czaja, D. J. Young, M. Kawecki, and R. M. Brown, Jr., Biomacromolecules 8, 1 (2007).

${ }^{24}$ J. Wang, Y. Zhu, and J. Du, J. Mech. Med. Biol. 11, 285 (2011).

${ }^{25}$ D. Zhang and L. Qi, Chem. Commun. 2005, 2735.

${ }^{26}$ H. S. Barud, T. Regiani, R. F. C. Marques, W. R. Lustri, Y. Messaddeq, and S. J. L. Ribeiro, J. Nanomater. 2011, 1.

${ }^{27}$ D. Klemm, D. Schumann, U. Udhardt, and S. Marsch, Prog. Polym. Sci. 26, 1561 (2001)

${ }^{28}$ W. K. Czaja, A. Krystynowic, S. Bielecki, and R. M. Brown, Jr., Biomaterials 27, 145 (2006).

${ }^{29}$ H. S. Barud, R. M. N. Assunção, M. A. U. Martines, J. Dexpert-Ghys, R. F. C. Marques, Y. Messaddeq, and S. J. L. Ribeiro, J. Sol-Gel Sci. Technol. 46, 363 (2008).

${ }^{30}$ A. M. Brito-Silva, A. Galembeck, A. S. L. Gomes, A. J. Jesus-Silva, and C. B. de Araújo, J. Appl. Phys. 108, 033508 (2010).

${ }^{31}$ I. Sondi and B. Salopek-Sondi, J. Colloid Interface Sci. 275, 177 (2004).

${ }^{32}$ J. S. Kim, E. Kuk, K. N. Yu, J.-H. Kim, S. J. Park, H. J. Lee, S. H. Kim, Y. K. Park, Y. H. Park, C.-Y. Hwang, Y.-K. Kim, Y.-S. Lee, D. H. Jeong, and M.-H. Cho, Nanomedicine 3, 95 (2007).

${ }^{33}$ X. Meng, K. Fujita, S. Murai, and K. Tanaka, Phys. Rev. A 79, 053817 (2009).

${ }^{34}$ X. Meng, K. Fujita, Y. Zong, S. Murai, and K. Tanaka, Appl. Phys. Lett. 92, 201112 (2008).

${ }^{35}$ S. Chen, X. Zhao, Y. Wang, J. Shi, and D. Liu, App. Phys. Lett. 101, 123508 (2012).

${ }^{36}$ B. Redding, M. A. Choma, and H. Cao, Nature Photon. 6, 355 (2012). 